\title{
La percepción de los estudiantes universitarios en relación con el trabajo interdisciplinario
}

\section{The perception of university students in relation to interdisciplinary work.}

\author{
RODRÍGUEZ-TORRES, Ángel F. ${ }^{1}$ \\ NARANJO-PINTO, Jorge A. ${ }^{2}$ \\ CARGUA-GARCÍA, Nancy I. ${ }^{3}$ \\ BUSTAMANTE-TORRES, Johana P. ${ }^{4}$ \\ CHASI-SOLORZANO, Byron F. ${ }^{5}$
}

\begin{abstract}
Resumen
El objetivo del estudio es analizar las percepciones de los estudiantes universitarios en relación con el trabajo interdisciplinario. La metodología tiene un enfoque cuantitativo de corte descriptivo y correlacional. Se administró el cuestionario a 581 estudiantes. Los resultados muestran que el trabajo colaborativo es lo más valorado; el mayor nivel de asociación se da entre la resolución de problemas-investigación; y existe diferencias significativas en las habilidades interdisciplinarias, actitudes reflexivas y trabajo colaborativo.

Palabras clave: interdisciplinariedad, educación superior, estudiantes universitarios.
\end{abstract}

\section{Abstract}

The objective is to analyze the perceptions of university students in relation to interdisciplinary work. The methodology has a quantitative approach of descriptive and correlational cut. The questionnaire was administered to 581 students. The results show that collaborative work is the most valued; the highest level of association is between problem solving-research; and there are significant differences in interdisciplinary skills, reflective attitudes and collaborative work

Key words: interdisciplinarity, higher education, university students.

\section{Introducción}

Los fenómenos que ocurren en la naturaleza, sociedad o pensamiento han rebasado la frontera de las disciplinas, por lo que es necesario evolucionar y avanzar en un abordaje interdisciplinario que responda a los problemas complejos que enfrenta la sociedad. Las disciplinas por sí solas ofrecen una visión egocéntrica y técnica de su especialidad, sin ninguna articulación con las demás disciplinas, respuestas o soluciones vagas a los problemas fundamentales que enfrenta la humanidad (Carvajal, 2010).

\footnotetext{
${ }^{1}$ Docente y Director de Posgrado de la Facultad de Cultura Física. Universidad Central del Ecuador. Ecuador. afrodriguez@uce.edu.ec

2 Docente de la Carrera de Psicopedadogía. Facultad de Filosofía, Letras y Ciencias de la Educación. Universidad Central del Ecuador. Ecuador. janaranjop@uce.edu.ec

${ }^{3}$ Docente de la Carrera Psicología Clínica de la Facultad de Ciencias Psicológicas. Universidad Central del Ecuador. Ecuador. ncargua@uce.edu.ec

${ }^{4}$ Docente de la Carrera de Psicopedadogía. Facultad de Filosofía, Letras y Ciencias de la Educación Universidad Central del Ecuador. Ecuador. jpbustamante@uce.edu.ec

${ }^{5}$ Docente de la Carrera de Psicopedadogía. Facultad de Filosofía, Letras y Ciencias de la Educación. Universidad Central del Ecuador. Ecuador. bchasi@uce.edu.ec
} 
Los problemas son vistos a partir de una disciplina y no a partir de la interrelación de otras disciplinas, esto genera la fragmentación y atomización del conocimiento y no permite comprender el todo. Por lo que es necesario establecer las conexiones entre los saberes que han sido separados, fomentar la comprensión de la complejidad de sus vínculos que configuran las situaciones reales,construir operadores cognitivos capaces de reconectar disciplinas y áreas del conocimiento que le permita tener una mejor comprensión del problema y plantear soluciones creativas (Pérez, 2012; Da Conceicao, 2006; Morin, 2002).

Esto se evidencia también en la formación inicial del profesorado por lo que existe la necesidad de reformular los procesos de formación del profesorado, incorporando la interdisciplinariedad, de manera que los conocimientos vayan más allá de la transmisión de saberes especializados (Errázuriz \& Soto, 2003), para que los docentes sean capaces de entender la complejidad de situaciones y adaptarse creativamente a la velocidad del cambio y a la incertidumbre que le acompaña (Pérez, 2010). Por lo tanto la educación "habría de enseñar estrategias que le permitan enfrentar los riesgos, lo inesperado y lo incierto, y modificar su desarrollo en virtud de informaciones adquiridas en el camino" (Morin, 2015, p. 38); y dar respuesta a los desafíos del siglo XXI, que es formar seres humanos con ciencia y con conciencia, desarrollador de una cultura del ser, capaz de enfrentar los problemas de la sociedad y plantear soluciones creativas (Pupo, 2007).

La universidad debe repensar cómo educar y formar a la humanidad que está inmersa en una transformación social, económica y tecnológica, donde si bien los conocimientos científicos y técnicos son cada vez más sofisticados, también conllevan increíbles cegueras y desorientaciones sobre las consecuencias de estas transformaciones en la realidad humana que ellos en parte provocan y, al mismo tiempo, sobre los desafíos que el actual horizonte planetario presenta (Motta, 2002). En este sentido, la universidad debe cambiar sus formas de pensar, contribuir a humanizar lo humano, crear reales vínculos entre disciplinas, vincular los conocimientos, desarrollar nuevas competencias, responder al contexto en un mundo globalizado y crear aptitudes sólidas para pensar de manera crítica y reflexiva (López, 2004); con el propósito que los estudiantes aprendan a través de la construcción interdisciplinaria de saberes, esto involucra superar una visión fragmentada y reduccionista (Carlachiani, 2016; Rodríguez et al., 2016). Esto se logrará si se "reforma la enseñanza que conduzca a la reforma del pensamiento y la reforma del pensamiento conduzca a la reforma de la enseñanza" (Morín, 2001, p. 23).

\subsection{Revisión de la literatura}

La interdisciplinariedad aparece como un constructo polisémico, mismo que para Morin (2002) es un proceso complejo e inacabado, una filosofía de trabajo que se nutre de un tejido de eventos, acciones, interacciones, determinaciones y azares que constituyen nuestro mundo fenoménico. Es decir, es una nueva forma de pensar y proceder, una filosofía de trabajo que involucra procesos caracterizados por su aval científico, sistemático e integrador de disciplinas, cuyo fin es resolver problemas complejos de la realidad mediante la creación de soluciones integrales, pertinentes, sostenibles y sustentables en una diversidad de escenarios.

Segovia et al. (2017) definen a la interdisciplinariedad como un "grupo de disciplinas relacionadas entre sí y con vínculos previamente establecidos, que evitan que se desarrollen acciones de forma aislada, dispersa o segmentada. Se trata de un proceso dinámico que pretende hallar soluciones a diferentes dificultades de investigación" (p. 83). De igual manera, a la intedisciplinariedad se la puede concebir como un proceso institucionalizado, dinámico, interactivo, recurrente, flexible, participativo e intencional que integra ciencias, áreas de conocimiento y disciplinas diversas que contribuya en la formación de profesionales a través del trabajo colaborativo, cooperativo, convergente que les posibiliten el planteamiento y generación de soluciones integrales, pertinentes, sostenibles, sustentables a problemas complejos del pensamiento, sociedad y naturaleza. En este sentido, se la puede enmarcar como un acto de intercambio, de reciprocidad, de convergencia, de coordinación y armonización para centrarse en la complementariedad entre diferentes puntos de vista de las disciplinas o ciencias para una lectura más amplia de la realidad (Ferreira, 2015). 
Es fundamental, en la formación de los futuros docentes, se promuevan experiencias apoyadas en la interdisciplinariedad que ayude a los estudiantes a tener una mejor comprensión y sentido a la ciencia con respecto a conceptos científicos abstractos y la función de la ciencia en la sociedad moderna, esto les permite tener una visión holística de los fenómenos del mundo real y de los problemas científicos y de su profesión (Sun, 2017); desarrollar habilidades metacognitivas, el pensamiento crítico y sistemático (Ivanitskaya et al., 2002), competencias reflexivas para el análisis y toma de decisiones durante el desarrollo de la práctica, pudiendo de esta manera vincular la teoría-práctica, el trabajo autónomo, cooperativo y colaborativo (Lizalde et al., 2019; Chacón et al., 2012); y, la investigación para crear conexiones entre disciplinas para la resolución de problemas complejos (de su vida personal y profesional) considerando el todo y no solo las partes (Adams et al, 2018, Rodríguez-Torres et al., 2020); esto se logra a través de actividades situadas (in situ) y auténticas, que le permiten aprender haciendo y transfir lo aprendido (aplicando modelos explicativos y conceptos aprendidos) de manera reflexiva a nuevas situaciones contribuyendo de esta manera a la mejora de su desempeño académico y un aprendizaje auténtico (You, 2017). Estas experiencias deben ser diseñadas por el profesorado en función de las habilidades y conceptos que los alumnos están aprendiendo en su curso académico; para lo cual debe coordinar las tareas y trabajos académicos que sean una oportunidad de aprendizaje práctico o experencial para crear una oportunidad de aprendizaje integrada y cohesionada para los estudiantes (Goldemberg et al., 2021).

En la práctica escolar, la interdisciplinariedad es una estrategia de acción donde la integración es el criterio que establece la cooperación entre partes con beneficio mutuo. Para la implementación de prácticas interdisciplinarias en la institución el profesorado es necesario: 1) percibirse a sí mismo como interdisciplinario; 2) tener la actitud o comportamiento interdisciplinario significa la reconstrucción de la institución volver a significar la acción educativa en sí misma para hacerla más eficiente y consistente; y, 3) intercambiar con cooperación mutua entre los actores educativos (Maris, 2000).

En esta línea, la necesidad de que la interdisciplinariedad es una necesidad para integrar los saberes y formar los futuros profesionales de la educación donde se considere los conocimientos disciplinarios, habilidades interdisciplinarias y actitudes reflexivas, así como la incorporación de estrategias metodológicas enfocadas en el trabajo colaborativo, el trabajo cooperativo, la investigación y a la resolución de problemas (Rodríguez-Santana, 2016). Esto demanda una reforma de la Universidad, es decir una reforma a la enseñanza presupone una reforma de pensamiento, donde ha primado una concepción tradicional fundamentada en la transmisión expositiva de conocimientos; por lo que es importante que aproveche las nuevas tecnologías y desarrolle habilidades interdisciplinares que preparen a los estudiantes para la amplia variedad de trabajos que probablemente realizarán después de graduarse, a través de la generación de vías de conexión entre la universidad y el trabajo, brindándoles las habilidades fundamentales y los conocimientos necesarios para convertirse en pensadores abiertos e interdisciplinarios que desafíen los paradigmas establecidos y las circunscripciones existentes (Goldemberg et al., 2021).

El objetivo principal que se plantea es analizar las percepciones de estudiantes universitarios en relación con el trabajo interdisciplinario. Este objetivo general conduce al planteamiento de los siguientes objetivos específicos, los mismos que orientan el estudio:

1. Analizar desde una perspectiva descriptiva las diversas dimensiones (conocimientos interdisciplinarios, habilidades interdisciplinarias, actitudes reflexivas, trabajo colaborativo, trabajo cooperativo, investigación y resolución de problemas) vinculadas al trabajo interdisciplinario.

2. Averiguar la relación existente y el nivel de correlación entre las dimensiones establecidas del trabajo interdisciplinario.

3. Descubrir la existencia de diferencias significativas en cada una de las dimensiones a nivel de género. 


\section{Metodología}

\subsection{Diseño de investigación y análisis de datos}

La investigación es un estudio ex posfacto, de enfoque cuantitativo de corte descriptivo y correlacional, ya que no se consideró el control de las variables, ni se influenció sobre ellas porque ya sucedieron, igual que sus efectos, fueron medidas en un determinado contexto y analizadas mediante métodos estadísticos (Hernández et al, 2016).

El análisis estadístico se efectuó con el programa IBM SPSS versión 25. En un primer momento se comprobaron los supuestos de linealidad, independencia, normalidad, homocedasticidad, análisis de residuos y no colinealidad, con la finalidad de determinar la validez de la aplicación del modelo estadístico. En base a lo revelado por los supuestos, se optó por utilizar pruebas paramétricas (Montilla \& Kromrey, 2010).

Se llevó a cabo un estudio descriptivo para revelar la distribución de las variables, empleando estadísticos como la media (M), el error estándar de medida (SEM), la desviación estándar (DE), coeficiente de variación (CV), la asimetría (Skw) y la curtosis (Kme). Asimismo, se realizaron correlaciones bivariadas de Pearson con el propósito de reflejar significancia estadística en la asociación lineal entre las distintas variables, así como su fuerza y dirección. A continuación, se calculó la prueba T de Student, como medida descriptiva para la comparación de medias, para comprobar la existencia de diferencias estadísticamente significativas.

\subsection{Participantes}

Participaron en el estudio 581 estudiantes de las carreras de Pedagogía de la Actividad Física y Deporte de la Facultad de Cultura Física y Psicología Educativa y Orientación de la Facultad de Cultura Física y Filosofía, Letras y Ciencias de la Educación de la Universidad Central del Ecuador, ubicada en la ciudad de Quito, de los cuales el $59,4 \%$ son hombres y el resto mujeres. Los estudiantes están distribuidos de la siguiente manera: 185 (31,8\%) de la carrera de Psicología Educativa y Orientación y 396 (68,2\%) de la carrera de Pedagogía de la Actividad Física y Deporte.

\subsection{Instrumento}

Para la construcción del instrumentos se realizó una revisión bibliográfica sobre la temática objeto de estudio. El cuestionario está constituido por 48 ítems, que consideraron siete (7) dimensiones: conocimientos interdisciplinarios (4 ítems), habilidades interdisciplinarias (14 ítems), actitudes reflexivas (5 ítems), trabajo colaborativo (9 ítems), trabajo cooperativo ( 7 ítems), investigación (4 ítems) y resolución de problemas (5 ítems). La valoración se hizo mediante una escala tipo Likert que consideró los valores de 1 a 5 , con las cualificaciones de: Totalmente de acuerdo (5), de acuerdo (4), ni de acuerdo ni en desacuerdo (3), en desacuerdo (2) y totalmente en desacuerdo (1).

La validación del cuestionario se realizó con la participación de profesionales expertos en las áreas de educación e investigación, con experiencia en la temática. De igual manera se realizó una prueba piloto con la participación de 84 estudiantes que no eran parte de la población investigada, obteniendo el Alfa de Cronbach $(\alpha=0,940)$ que representa una alta fiabilidad de los datos.

Al inicio del cuestionariose se incluyó un párrafo mediante el cual se informó a los estudiantes sobre el objetivo de la investigación, el manejo de la confidencialidad de los datos, la libre y voluntaria participación (consentimiento), así como la inexistencia de riesgos y compromisos. 


\subsection{Procedimiento para la aplicación del instrumento}

Se contó con la autorización de las autoridades de las carreras. Para la recopilación de información se aplicó el cuestionario en línea con la herramienta Formularios de Google. Una vez recopilada toda la información se produjo el análisis estadístico, que posibilitó establecer hallazgos como respuesta a los objetivos de la investigación formulados.

Para la obtención del aval ético, se presentó la documentación requerida al Subcomité de Ética de la Universidad Central del Ecuador, el cual después de valorarlo emitió su criterio, por lo que la investigación se realizó respetando las normas legales vigentes y los criterios éticos establecidos.

\section{Resultados y discusión}

\subsection{Analisis descriptivos}

Atendiendo a los datos alcanzados en el análisis estadístico, se puede indicar que la media de valoración se sitúa muy por encima de la media totalizada, ubicada en 3. Esto muestra una valoración muy positiva por parte de los estudiantes en relación con el trabajo interdisciplinario. Todas las medias se sitúan por encima de 3 y, de todas las dimensiones, la más valorada es el trabajo colaborativo y la que menos media presenta es habilidades interdisciplinarias. Siguiendo con el análisis, y teniendo presente lo establecido por Jöreskog (2001), la distribución de los datos es normal, dado que los valores de asimetría se sitúan entre -1.96 y +1.96 . Esto viene a justificar los diversos análisis estadísticos que se desarrollan a posterior. Es decir, justifica el uso de pruebas paramétricas. Además, el uso de pruebas paramétricas viene reforzado por los valores obtenidos en el coeficiente de varianza, los cuales se ubican por debajo de 0.2 . La curtosis de todas las dimensiones es variable leptocúrtica (tabla 1).

Tabla 1

Estadísticos descriptivos dimensiones sobre

el trabajo interdisciplinario en la universidad.

\begin{tabular}{|c|c|c|c|c|c|c|}
\hline DIMENSIONES & $\mathbf{M}$ & $\mathbf{E T M}$ & $\mathbf{D T}$ & $\mathbf{C V}$ & $\mathbf{S}_{\mathbf{k w}}$ & $\mathbf{K}_{\mathbf{m e}}$ \\
\hline $\begin{array}{c}\text { Conocimientos } \\
\text { disciplinarios }\end{array}$ & 4,1519 &, 02260 &, 54482 &, 131 &,- 780 & 1,537 \\
\hline $\begin{array}{c}\text { Habilidades } \\
\text { interdisciplinares }\end{array}$ & 4,1210 &, 02283 &, 55030 &, 134 &,- 857 & 1,344 \\
\hline Actitudes reflexivas & 4,3415 &, 02217 &, 53435 &, 123 &,- 952 & 1,489 \\
\hline $\begin{array}{c}\text { Trabajo } \\
\text { colaborativo }\end{array}$ & 4,4483 &, 02048 &, 49358 &, 111 & $-1,306$ & 2,734 \\
\hline $\begin{array}{c}\text { Trabajo } \\
\text { cooperativo }\end{array}$ & 4,2011 &, 02525 &, 60871 &, 145 &,- 754 &, 600 \\
\hline $\begin{array}{c}\text { Investigación } \\
\text { Resolución de } \\
\text { problemas }\end{array}$ & 4,1910 &, 02875 &, 69290 &, 165 &,- 767 &, 272 \\
\hline
\end{tabular}

Nota: $M=$ =media; ETM=Error típico de la media; DT=Desviación típica;

$\mathrm{CV}=$ Coeficiente de varianza; $\mathrm{S}_{\mathrm{kw}}=$ Asímetría; $\mathrm{K}_{\mathrm{me}}=$ curtosis

\subsection{Correlación entre dimensiones}

Las correlaciones establecidas entre las diversas dimensiones de estudio muestran una relación significativa entre todas ellas. La fuerza de asociación establecida es media en la mayoría de los casos, habiendo dimensiones con una fuerza de asociación media-alta. Esto indica que todas las dimensiones aplicadas en el estudio están relacionadas entre sí, lo que refuerza que la predisposición hacia el trabajo interdisciplinario en los procesos de 
enseñanza y aprendizaje genera mejora en todas las dimensiones. Las dimensiones donde el valor de asociación es más alto es la resolución de problemas-investigación. De estos datos se puede determinar que hay una influencia directa entre la resolución de problemas por parte de los estudiantes a partir de la aplicación de la investigación. En cambio, las dimensiones con menor fuerza de asociación es la investigación y el trababjo colaborativo, donde los valores se sitúan entorno a 0.408 (tabla 2).

Tabla 2

Correlaciones entre dimensiones

\begin{tabular}{|c|c|c|c|c|c|c|}
\hline \multicolumn{7}{|c|}{ Correlaciones } \\
\hline $\begin{array}{c}\text { Dimensiones } \\
\text { Conocimientos } \\
\text { disciplinarios }\end{array}$ & $\begin{array}{c}\text { Conocimientos } \\
\text { disciplinarios }\end{array}$ & $\begin{array}{c}\text { Habilidades } \\
\text { Interdisciplinarias }\end{array}$ & $\begin{array}{c}\text { Actitudes } \\
\text { Reflexivas }\end{array}$ & $\begin{array}{c}\text { Trabajo } \\
\text { colaborativo }\end{array}$ & $\begin{array}{c}\text { Trabajo } \\
\text { cooperativo }\end{array}$ & $\begin{array}{c}\text { Resolver } \\
\text { Investigación } \\
\text { problemas }\end{array}$ \\
\hline $\begin{array}{c}\text { Habilidades } \\
\text { Interdisciplinarias }\end{array}$ &, $653^{* *}$ & 1,000 & & & & \\
\hline $\begin{array}{c}\text { Actitudes } \\
\text { Reflexivas }\end{array}$ &, $537^{* *}$ &, $622^{* *}$ & 1,000 & & & \\
\hline $\begin{array}{c}\text { Trabajo } \\
\text { colaborativo }\end{array}$ &, $425^{* *}$ &, $503^{* *}$ &, $620^{* *}$ & 1,000 & & \\
\hline $\begin{array}{c}\text { Trabajo } \\
\text { cooperativo }\end{array}$ &, $529^{* *}$ &, $486^{* *}$ &, $522^{* *}$ &, $572^{* *}$ & 1,000 & \\
\hline $\begin{array}{c}\text { Investigación } \\
\text { Resolver } \\
\text { problemas }\end{array}$ &, $443^{* *}$ &, $420^{* *}$ &, $453^{* *}$ &, $408^{* *}$ &, $670^{* *}$ & 1,000 \\
\hline
\end{tabular}

Elaboración propia **. La correlación es significativa en el nivel 0,01 (bilateral)

\subsection{Diferencias significativas entre dimensiones a nivel de género}

Tabla 3

Resultados diferenciados según el género de los docentes en cada una de las dimensiones de estudios.

\begin{tabular}{|c|c|c|c|c|c|c|}
\hline \multicolumn{7}{|c|}{ GÉNERO } \\
\hline Dimensiones & Género & $n$ & $M$ & DT & $\mathrm{t}$ & $p$ \\
\hline \multirow{2}{*}{$\begin{array}{l}\text { Conocimientos } \\
\text { disciplinares }\end{array}$} & $\mathrm{H}$ & 236 & 4,1155 & ,52911 & \multirow[t]{2}{*}{$-1,334$} & \multirow[t]{2}{*}{ 183 } \\
\hline & $M$ & 345 & 4,1768 & ,55468 & & \\
\hline \multirow{2}{*}{$\begin{array}{c}\text { Habilidades } \\
\text { interdisciplinares }\end{array}$} & $\mathrm{H}$ & 236 & 4,0518 & ,55393 & \multirow[t]{2}{*}{$-2,519$} & \multirow[t]{2}{*}{012} \\
\hline & $\mathrm{M}$ & 345 & 4,1683 &, 54354 & & \\
\hline \multirow[t]{2}{*}{ Actitudes reflexivas } & $\mathrm{H}$ & 236 & 4,4017 & ,54045 & \multirow[t]{2}{*}{2,254} & \multirow[t]{2}{*}{,025 } \\
\hline & $\mathrm{M}$ & 345 & 4,3003 & ,52698 & & \\
\hline \multirow{2}{*}{$\begin{array}{c}\text { Trabajo } \\
\text { colaborativo }\end{array}$} & $\mathrm{H}$ & 236 & 4,5198 & ,44453 & \multirow[t]{2}{*}{2,907} & \multirow[t]{2}{*}{,004 } \\
\hline & $\mathrm{M}$ & 345 & 4,3994 & ,51947 & & \\
\hline \multirow{2}{*}{$\begin{array}{c}\text { Trabajo } \\
\text { cooperativo }\end{array}$} & $\mathrm{H}$ & 236 & 4,1816 & ,64353 & \multirow[t]{2}{*}{,- 639} & \multirow[t]{2}{*}{523} \\
\hline & $M$ & 345 & 4,2145 & ,58427 & & \\
\hline \multirow{2}{*}{ Investigación } & $\mathrm{H}$ & 236 & 4,1582 & ,76286 & \multirow[t]{2}{*}{,- 945} & \multirow[t]{2}{*}{,345 } \\
\hline & $\mathrm{M}$ & 345 & 4,2135 & ,64083 & & \\
\hline \multirow{2}{*}{$\begin{array}{c}\text { Resolución } \\
\text { de problemas }\end{array}$} & $\mathrm{H}$ & 236 & 4,1136 & ,70595 & \multirow[t]{2}{*}{$-1,132$} & \multirow[t]{2}{*}{,258 } \\
\hline & $M$ & 345 & 4,1745 & ,58592 & & \\
\hline
\end{tabular}

Nota: H: Hombre; M: Mujer; n=muestra; M=media; DT=Desviación típica; $t=$ T de Student; $p=p$ valor. 
En la tabla 3 se representa la relación establecida entre el género con las diversas dimensiones de estudio. Las dimensiones mejor valoradas son el trabajo colaborativo y actitudes reflexivas por hombres y mujeres. Se aplicó la T de Student para muestras independientes en el caso de las variables de género. Los resultados estadísticos obtenidos muestran que sí hay diferencias de valoración y que son significativas $(p<0.05)$ en las siguientes dimensiones de estudio: habilidades interdisciplinares, actitudes reflexivas y trabajo colaborativo (tabla 3).

\subsection{Discusión}

Las instituciones de educación superior tienen la responsabilidad, ante la sociedad, de formar de manera integral al futuro profesional, que tenga la capacidad de integrar sus saberes disciplinares y pedagógicos, para que logren una mejor comprensión de su contexto y resuelvan, de manera creativa y colaborativa, los problemas que enfrentará en la vida real y en su profesión (Rodríguez-Torres et al, 2018; Fullan, \& Langworthy, 2014).

En este sentido, el docente juega un rol fundamental ya que debe generar un ambiente favorable en el aula, para que el estudiante realice el intercambio interdisciplinar, vinculando el aprendizaje entre materias y entre contextos. Esto implica la construcción de competencias en sus estudiantes que les permitan integrar sus saberes no solo en la universidad, sino por lo contrario en su vida personal y profesional (Taylor, 2016).

A continuación, se muestran los aspectos más relevantes alcanzados en los resultados:

En función de los objetivos planteados en el estudio se acompañan los comentarios relacionados con la interpretación de los resultados obtenidos:

- Objetivo 1: Analizar desde una perspectiva descriptiva las diversas dimensiones (conocimientos interdisciplinarios, habilidades interdisciplinarias, actitudes reflexivas, trabajo colaborativo, trabajo cooperativo, investigación y resolución de problemas) vinculadas al trabajo interdisciplinario.

La media de valoración se sitúa alrededor de 4 en todos los casos, lo que indica que la valoración media de los estudiantes de cara al trabajo interdisciplinario es alta por su importancia en el aprendizaje del alumnado.

La dimensión mejor valorada es el trabajo colaborativo, esto se debe a que los diseños curriculares fomentan estrategias metodológicas que propician un aprendizaje activo y responsable en el estudiante a través de la creación de comunidades profesionales de aprendizaje, donde se promueva el dialogo y la reflexión de la práctica docente, en el que todos sus integrantes contribuyen con su interacción a la resolución problemas de su profesión, que favorecen la formación de los futuros docentes (Fombona et al, 2016) y tienen la posibilidad de incorporar otro tipo de aprendizajes más colaborativos (Silva-Peña, \& Salgado, 2013). La implementación de metodologías innovadoras permite integrar los saberes disciplinares y pedagógicos (Marín-González et al, 2020), se garantiza en el alumnado el éxito en su proceso de aprendizaje y desarrollo personal (Real et al, 2020), desarrollan habilidades interpersonales, conocimiento disciplinario más profundo y comprensión amplia de otras disciplinas y se siente mejor preparado para ingresar al trabajo (Warr, \& West, 2020).

La dimensión menos valorada son las habilidades interdisciplinares, esto se debe a que en las carreras los maestros enseñan una disciplina específica lo que dificulta la implementación de la enseñanza interdisciplinaria debido a la falta de preparación y experiencias concretas. Si los profesores no tienen solvencia en los temas específicos de su disciplina tendrán dificultad en integrar los conceptos, lo que repercute en que los estudiantes carezcan de una visión holística de la ciencia y limita su capacidad de aprender ciencia relacionada con la vida real (Sun, 2017). De esta manera no puedan usar las diferentes perspectivas disciplinarias, hacer conexiones e integrar sus saberes en la resolución de problemas (Brassler, \& Dettmers, 2017).

La distribución de datos es normal, lo que indica que existe una percepción conjunta del profesorado. Es decir, la mayoría opina de manera similar en cuanto a la aplicación de métodos pedagógicos innovadores. 
- Objetivo 2: Averiguar la relación existente y el nivel de correlación entre las dimensiones establecidas del trabajo interdisciplinario.

Las dimensiones del estudio están relacionadas entre sí, lo que refuerza la predisposición hacia el trabajo interdisciplinario en los procesos de enseñanza y aprendizaje generando mejora en todas las dimensiones.

Las dimensiones donde el valor de asociación es más alto son en la investigación y la resolución de problemas. De estos datos se puede determinar que hay una influencia directa entre la investigación que realizan los estudiantes durante su proceso de formación y la resolución de problemas de su profesión, donde el profesorado implemente estrategias que fomenten la indagación en el aula y los estudiantes gestionen su propio aprendizaje para construir un conocimiento auténtico y luego puedan aplicarlo o transferirlo en la vida cotidiana, en nuevos contextos y en la resolución de problemas complejos (Rodríguez-Gallego, \& Ordóñez-Sierra, 2021; RodríguezTorres et al, 2018; Rodríguez, \& Naranjo, 2016). Lo que es visto por los estudiantes como una opción de mejora, aprendizaje y valor para la investigación (Calisto-Alegría, 2021).

En cambio, las dimensiones con menor fuerza de asociación son la investigación y las habilidades interdisciplinarios.

Existe la predisposición de los estudiantes en desarrollar las siguientes dimensiones como: habilidades interdisciplinares, actitudes reflexivas y el trabajo colaborativo en su proceso de formación. En este caso, se observa que las dimensiones mejor valoradas son el trabajo colaborativo y actitudes reflexivas, concretamente los hombres en relación con las mujeres.

Esto evidencia que los estudiantes han desarrollado habilidades interdisciplinarias que les permite evaluar las distintas perspectivas disciplinares, el reconocimiento de las limitaciones de cada disciplina y la manera de aplicarla de forma manera interdisciplinaria en su formación, la habilidad para encontrar terrenos comunes entre disciplinas (Hernández-Armenta, \& Domínguez, 2019) y actitudes reflexivas que los llevan a integrar holísticamente aquellos conocimientos y modos de pensar de dos o más disciplinas para crear productos, resolver problemas complejos y ofrecer explicaciones del mundo que los rodea, en formas que no habrían sido posibles a través de medios disciplinarios únicos (Boix, Miller y Gardner, 2000). Esto se logra a través de metodologías innovadoras que fomenten la colaboración, cooperación, complementación e integración entre disciplinas, evaluación y en las formas de organizar entornos para el aprendizaje (González \& Iglesias, 2016; Da Silva et al, 2018). En el resto de las dimensiones no se observan diferencias significativas.

\section{Conclusiones}

Se puede concluir que los estudiantes valoran positivamente al trabajo colaborativo como una estrategia innovadora empleada en el aula y su práctica ofrece un escenario para la construcción colectiva del conocimiento que le permite, tanto el desarrollo de habilidades sociales y comunicativas, como también la resolución de problemas. Existe una mayor relación entre la investigación-resolución de problemas, esto se debe a que el profesorado implementa en el aula estrategias que fomentan la indagación, lo que facilita que los estudiantes aprendan a dentificar y plantear preguntas, buscar información pertinente y actualizada, diseñar y realizar investigaciones, recolectar los datos, analizar datos y pruebas, utilizar modelos y explicaciones y diversas maneras de comunicar los hallazgos. Lo que le permite tener una mejor comprensión del objeto de estudio y resolver problemas complejos que se presentan en su vida cotidiana y en el ejercicio de su profesión. En lo referente al género las dimensiones mejor valoradas son el trabajo colaborativo y actitudes reflexivas, por los hombres y las mujeres. Pero existe una diferencia significativa entre hombres y mujeres en las siguientes dimensiones: habilidades interdisciplinares, actitudes reflexivas y el trabajo colaborativo. 
Las limitaciones de esta investigación se circunscriben a solo un grupo de estudiantes debido a que debido a la pandemia COVID 19 no fue factible recolectar datos a una mayor cantidad de estudiantes de ambas carreras. Por otra parte, el estudio se realizó con estudiantes de dos carreras, pero para un posterior estudio se pueden abarcar otras carreras que permitan tener una visión global en relación con el objeto de estudio. En cuanto a futuras líneas de investigación se debe afirmar que sería conveniente y necesario realizar un estudio sobre la interdisciplinariedad en las universidades del país que sería altamente beneficioso conocer datos de este tipo.

\subsection{Implicaciones del estudio}

Este estudio es una respuesta a la carencia de datos en el Ecuador en lo referente a la percepción del alumnado en relación con el trabajo interdisciplinario y su impacto en su formación profesional. Los resultados alcanzados en esta investigación han dado origen a diversas implicaciones de carácter teórico y práctico. A nivel teórico, el presente trabajo contribuye al aumento de la literatura científica sobre la interdisciplinariedad. Además, los hallazgos obtenidos han permitido determinar que es necesario que el profesorado implemente estrategias innovadoras que fomenten la indagación en el aula. Esto permite orientar a la comunidad científica sobre los principales focos de interés que los investigadores han ido tomando durante su producción científica y hacia donde tiende la investigación.

Asimismo, los resultados han permitido revelar que los estudiantes valoran positivamente la dimensión de trabajo colaborativo y que existe una mayor relación entre la investigación y la resolución de problemas. Estos pueden servir de guía y sustento para futuros trabajos por parte de otros científicos que requieran información sobre el trabajo interdisciplinario en la educación superior en un país latinoamericano.

Por otro lado, este estudio deja una serie de implicaciones prácticas de interés para diversos colectivos relacionados con la innovación educativa en instituciones de educación superior. Esto abarca desde profesores, investigadores, estudiantes, hasta entidades e instituciones encargadas de la formación del profesorado. Por último, los resultados obtenidos pueden servir de guía para las entidades e instituciones encargadas de la planificación y desarrollo de la formación del profesorado. Todo ello con el propósito de conocer la realidad educativa en lo referente a la interdisciplinariedad y desarrollar propuestas que contribuyan a su desarrollo e integración en los procesos formativos.

\subsection{Agradecimientos}

Los investigadores agradecen a la Universidad Central del Ecuador que, por medio de la Dirección de Investigación, contribuye con esta investigación "La interdisciplinariedad como estrategia para la formación de profesionales de la Cultura Física y Psicología Educativa", CÓDIGO: DICONV-2019-069, con financiamiento y la asignación de carga horaria para los investigadores, cuyos resultados parciales se presentan en este artículo.

\section{Referencias bibliográficas}

Adams, S., Brown, M., Dahlstrom, E., Davis, A., DePaul, K., Diaz, V., \& Pomerantz. J. (2018). NMC Horizon Report: 2018 Higher Education Edition. EDUCAUSE.

Boix, V., Miller, W. C., \& Gardner, H. (2000.) On disciplinary lenses and interdisciplinary work. In S. Wineburg \& P. Grossman (Eds.), Interdisciplinary curriculum: Challenges to implementation. Teachers College Press.

Brassler, M., \& Dettmers, J. (2017). How to Enhance Interdisciplinary Competence-Interdisciplinary ProblemBased Learning versus Interdisciplinary Project-Based Learning. Interdisciplinary Journal of Problem-Based Learning, 11(2), 1-15. https://doi.org/10.7771/1541-5015.1686 
Calisto-Alegría, C. (2021). Adquisición de habilidades investigativas de los profesores en formación en Seminario de Grado. Revista Complutense de Educación, 32(2), 205-215. https://doi.org/10.5209/rced.68317

Carlachiani, C. (2016). Los núcleos interdisciplinarios de contenidos (NIC) y el acontecimiento. En S. Morelli (Coord.). Núcleos Interdisciplinarios de Contenido NIC. (pp. 23-38). Rosario: HomoSapiens

Carvajal, Y. (2010). Interdisciplinariedad: Desafío para la educación superior y la investigación. Revista Luna Azul, 31, 156-169. https://doi.org/10.17151/luaz.2010.31.12

Chacón, M., Chacón, C., \& Alcedo, Y. (2012). Los proyectos de aprendizaje interdisciplinarios en la formación docente. Revista Mexicana de Investigación Educativa, 17 (54), 877-902.

Da Conceicao, M. (2006). Para comprender la complejidad. México: S/e.

Da Silva, A., Cusati, I., \& Guerra, M. (2018). Interdisciplinaridade e Transdisciplinaridade: Dos conhecimentos e suas historias. RIAEE - Revista Ibero-Americana de Estudos em Educação, Araraquara, 13(3), 979-996. https://doi.org/10.21723/riaee.v13.n3.2018.11257

Errázuriz, J. \& Soto, A. (2003). Aportes de un enfoque interdisciplinarios en la formacion inicial docente. Revista Electrónica Diálogos Educativos. 3(5), 62-73.

Ferreira, M. (2015). Ciencia e interdisciplinariedad. En I. Fazenda (Coord.). Prácticas interdisciplinares en la escuela. (pp. 23-28). Barcelona: Octaedro, S.L.

Fombona, J., Iglesias, M., \& Lozano, I. (2016). El trabajo colaborativo en la educación superior: una competencia profesional para los futuros docentes. Educ. Soc., Campinas, 37(135), 519-538. https://doi.org/10.1590/es0101-73302016147914

Fullan, M. \& Langworthy, M. (2014). Una rica veta. Cómo las nuevas pedagogías logran el aprendizaje en profundidad. PEARSON. Recuperado de https://www.pearson.com/content/dam/one-dot-com/one-dotcom/global/Files/about-pearson/innovation/open-ideas/ARichSeamSpanish.pdf

González, J., \& Iglesias, M. (2016). Concepción de una propuesta curricular interdisciplinaria: Una visión desde la asignatura de Bioquímica en el ciclo básico de la carrera de Medicina en la universidad de Guayaquil Ecuador. Revista Universidad y Sociedad, 8 (1), 7-12. https://doi.org/10.36576/summa.14332

Goldemberg, D., Genone, J., \& Wisor, Sc. (2021). ¿Cómo preparan los innovadores disruptivos a los estudiantes de hoy para ser la fuerza laboral del mañana? El modelo cooperativo un camino para cerrar la brecha de habilidades. Banco Interamericano de Desarrollo. Recuperado de https://publications.iadb.org/publications/spanish/document/Como-preparan-los-innovadoresdisruptivos-a-los-estudiantes-de-hoy-para-ser-la-fuerza-laboral-del-manana-El-modelo-cooperativo-deMinerva-Un-camino-para-cerrar-la-brecha-de-habilidades.pdf https://doi.org/10.18235/0003052

Hernández-Armenta, I., \& Domínguez, A. (2019). Evaluación de Percepciones Sobre la Interdisciplinariedad: Validación de Instrumento para Estudiantes de Educación Superior. Formación Universitaria, 12(3), 27-38. https://doi.org/10.4067/s0718-50062019000300027

Hernández, R., Fernández, C., \& Baptista, P. (2016). Metodología de la Investigación. (Quinta Edición). México D.F, México: McGraw-Hill

Ivanitskaya, L., Clark, D., Montgomery, G., \& Primeau, R. (2002). Interdisciplinary Learning: Process and Outcomes. Innovative Higher Education, 27(2), 95-111. https://doi.org/10.1023/a:1021105309984 
Jöreskog, K. (2001). Analysis of ordinal variables 2. Cross-sectional data. Taller "Structural Equation Modelling with LISREL 8.51". Friedrich-Schiller-Universitat, Jena.

Lizalde, M., Peñarrubia, C., Murillo, B., Latorre, J., \& Canales-Lacruz, I. (2019). Aprendizaje por proyectos e interdisciplinariedad en la mención de la educación física del grado de maestro de primaria. Retos, 35, 391-395. https://doi.org/10.47197/retos.v0i35.63446

López, G. (2004). Una mirada integral que permita descubrir el sentido y respuestas de la universidad en el futuro. Quito: Mariscal.

Marín-González, F., Grimaldo-Guerrero, J., Mendoza-Becerra, M., \& Senior-Naveda, A. (2020). Mapeo del conocimiento para la enseñanza interdisciplinaria en ingeniería. Formación Universitaria, 13(6), 85-94. https://doi.org/10.4067/s0718-50062020000600085

Maris, E. (2000). O "como" implementar a dimensão interdisciplinar em práticas pedagógocas nas escolas. Contexto e Educação, 15(60), 81-98.

Montilla, J., \& Kromrey, J. (2010). Robustez de las pruebas T en comparación de medias, ante violación de supuestos de normalidad y homocedasticidad. Ciencia e Ingeniería, 31, 101-108. https://doi.org/10.33262/cienciadigital.v2i4.1..187

Morin, E. (2015). Enseñar a vivir. Buenos Aires: Nueva Visión.

Morin, E. (2002). La cabeza bien puesta. Buenos Aires: Nueva Visión.

Morin, E. (2001). La mente bien ordenada. Barcelona: Seix Barral, S.A.

Motta, R. (2002). Complejidad, educación y transdisciplinariedad. Polis, 3, 1-16. Recuperado de http://polis.revues.org/7701

Pérez, Á. (2012). Educarse en la era digital. Madrid: Morata.

Pérez, Á. (2010). Aprender a educar. Nuevos desafíos para la formación de docente. Revista Interuniversitaria de Formación del Profesorado, 68 (24,2), 37-60. https://doi.org/10.47553/rifop.v34i2

Pupo, R. (2007). El ensayo como búsqueda y creación. (Hacia un discurso de aprehensión compleja). México: s/e.

Real, S., Ramírez, S., Bermúdez, M., \& Pino, A. (2020). Las metodologías empleadas en la innovación educativa. Aula de Encuentro, 22 (1), 57-80. https://doi.org/10.17561/ae.v22n1.3

Rodríguez-Gallego, M., \& Ordóñez-Sierra, R. (2021). Metodologías activas desarrolladas en la supervisión de las Prácticas Externas del Grado en Pedagogía. Revista d'Innovació Docent Universitària, 13, 1-8. https://doi.org/10.1344/ridu2019.11.3

Rodríguez, Á. \& Naranjo, J. (2016). El aprendizaje basado en problemas: una oportunidad para aprender. Lecturas Educación Física y Deportes. Buenos Aires, Año 21, No 221, Octubre. http://www.efdeportes.com/efd221/el-aprendizaje-basado-en-problemas.htm

Rodríguez, Á., Capote, G., Rendón, P. \& Valdés, C. (2016), El pensamiento complejo en el diseño curricular de la Educación Física. Lecturas Educación Física y Deportes. Buenos Aires, Año 21, No 215, Abril. http://www.efdeportes.com/efd215/el-pensamiento-complejo-en-la-educacionfisica.htm 
Rodríguez-Santana, S. (2016). La Interdisciplinaridad: Una vía para establecer la relación entre la filosofía y la enseñanza de la historia de Cuba. Educación y Sociedad, 14(1), 26- 39.

https://doi.org/10.15517/aie.v7i4.9301

Rodríguez-Torres, Á., Mendoza-Yépez , M., Cargua-García, N., \& Gudiño-Negrete, X. (2020). La interdisciplinariedad en la formación de profesionales de la Actividad Física y Deporte - Universidad Central del Ecuador. Polo del Conocimiento, 5(9), 1179-1199.

Rodríguez-Torres, Á., Fierro-Altamirano, R., Vela-Larco, D. \& Quijano-Rojas, M. (2018). La resolución de problemas: una oportunidad para aprender a aprender. Olimpia. Revista de la Facultad de Cultura Física de la Universidad de Granma, 15(50), 160-171. https://doi.org/10.17227/01212494.26pys57.66

Rodríguez-Torres, Á., Posso-Pacheco, R., De la Cueva-Constante, R. \& Barba-Miranda, L. (2018). Herramientas metodológicas para el desarrollo de habilidades investigativas en los estudiantes. Una praxis necesaria. Olimpia: Revista de la Facultad de Cultura Física de la Universidad de Granma, 15(50), 119-132.

Segovia, P., Pinos, P. \& Murillo, I. (2017). La interdisciplinariedad para el perfeccionamiento del proceso de enseñanza aprendizaje en la Carrera de Odontología. Revista Conrado, 13(57), 82-90. Recuperado de https://doi.org/10.37117/s.v2i3.23

Silva-Peña, I., \& Salgado, I. (2013). Uso de wikis como herramienta de trabajo colaborativo en un proceso de formación inicial docente. Ciencia, Docencia y Tecnología, 24(46), 165-179.

Sun, H. (2017). Why Teach Science with an Interdisciplinary Approach: History, Trends, and Conceptual Frameworks. Journal of Education and Learnin, 6(4), 66-77.

Taylor, M. (2016). Aprendizaje integrado en Educación superior en Estados Unidos. Donde estábamos y hacia donde nos dirigimos. En D. Blackshields, J. Cronin, B. Higgs, S. Kilcommis, M. McCarthy, \& A. Ryan (Coords.). Aprendizaje Integrado. Investigaciones internacionales y casos prácticos. (pp. 21-36). Madrid: Narcea. https://doi.org/10.2307/j.ctv1f70kjw.15

Warr, M., \& West, R. (2020). Bridging Academic Disciplines with Interdisciplinary Project-based Learning. The Interdisciplinary Journal of Problem-based Learning, 14(1), 1-22.

https://doi.org/10.14434/ijpbl.v14i1.28590

You, H. (2017). Why Teach Science with an Interdisciplinary Approach: History, Trends, and Conceptual Frameworks. Journal of Education and Learning. 6 (4), 66-77. https://doi.org/10.5539/jel.v6n4p66

Esta obra está bajo una Licencia Creative Commons Attribución-NoCommercial 4.0 International

(cc) EY-NC 